\title{
Comparison of the dose on specific 3DCT images and the accumulated dose for cardiac structures in esophageal tumors radiotherapy: whether specific 3DCT images can be used for dose assessment?
}

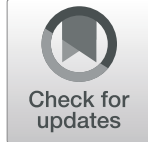

Ying Tong ${ }^{1}$, Guanzhong Gong ${ }^{1}$, Ming $\mathrm{Su}^{2}$ and Yong Yin ${ }^{1 *}$

\begin{abstract}
Background: Cardiac activity could impact the accuracy of dose assessment for the heart, pericardium and left ventricular myocardium (LVM). The purpose of this study was to explore whether it is possible to perform dose assessment by contouring the cardiac structures on specific three-dimensional computed tomography (3DCT) images to reduce the impact of cardiac activity.
\end{abstract}

Methods: Electrocardiograph-gated 4DCT (ECG-gated 4DCT) images of 22 patients in breath-hold were collected. MIM Maestro 6.8.2 (MIM) was used to reconstruct specific 3DCT images to obtain the Maximal intensity projection (MIP) image, Average intensity projection (AIP) image and Minimum intensity projection (Min-IP) image. The heart, pericardium and LVM were contoured in 20 phases of 4DCT images $(0,5 \% \ldots 95 \%)$ and the MIP, AIP and Min-IP images. Then, a radiotherapy plan was designed at the $0 \%$ phase of the 4DCT images, and the dose was transplanted to all phases of 4DCT to acquire the dose on all phases, the accumulated dose of all phases was calculated using MIM. The dose on MIP, AIP and Min-IP images were also obtained by deformable registration of the dose. The mean dose $\left(D_{\text {mean }}\right), V_{5}, V_{10}, V_{20}, V_{30}$ and $V_{40}$ for the heart, pericardium and LVM in MIP, AIP and Min-IP images were compared with the corresponding parameters after dose accumulation.

Results: The mean values of the difference between the $D_{\text {mean }}$ in the MIP image and the $D_{\text {mean }}$ after accumulation for the heart, pericardium and LVM were all less than $1.50 \mathrm{~Gy}$, and the dose difference for the pericardium and LVM was not statistically significant $(p>0.05)$. For dose-volume parameters, there was no statistically significant difference between $V_{5}, V_{10}$, and $V_{20}$ of the heart and pericardium in MIP, AIP, and Min-IP images and those after accumulation $(p>0.05)$. For the LVM, only in the MIP image, the differences of $V_{5}, V_{10}, V_{20}, V_{30}$ and $V_{40}$ were not significant compared to those after dose accumulation ( $p>0.05)$.

Conclusions: There was a smallest difference for the dosimetry parameters of cardiac structures on MIP image compared to corresponding parameters after dose accumulation. Therefore, it is recommended to use the MIP image for the delineation and dose assessment of cardiac structures in clinical practice.

Keywords: Esophageal tumors radiotherapy, Cardiac activity, Cardiac dose, Specific 3DCT images, Dose accumulation

\footnotetext{
* Correspondence: yinyongsd@126.com

'Shandong Cancer Hospital and Institute, Shandong First Medical University

and Shandong Academy of Medical Sciences, Jinan, China

Full list of author information is available at the end of the article
}

(c) The Author(s). 2019 Open Access This article is distributed under the terms of the Creative Commons Attribution 4.0 International License (http://creativecommons.org/licenses/by/4.0/), which permits unrestricted use, distribution, and reproduction in any medium, provided you give appropriate credit to the original author(s) and the source, provide a link to the Creative Commons license, and indicate if changes were made. The Creative Commons Public Domain Dedication waiver (http://creativecommons.org/publicdomain/zero/1.0/) applies to the data made available in this article, unless otherwise stated. 


\section{Introduction}

Radiotherapy plays an important role in the comprehensive treatment of thoracic tumors, such as esophageal cancer, breast cancer and lung cancer [1-4]. Madan et al. reported that more than 50\% of thoracic tumor patients require radiotherapy at some point in time [5]. Radiation therapy not only brings benefits to patients, but also damages to normal tissues [6, 7]. As the main complication of radiotherapy for thoracic tumors, radiation-induced heart disease (RIHD) has attracted much attention, including radiationinduced pericarditis, radiation-induced valvular disease, radiation-induced myocarditis, etc $[5,8,9]$ Therefore, the accuracy of radiotherapy for thoracic tumors is particularly important.

In the process of radiotherapy for thoracic tumors, one of the important factors affecting the accuracy of treatment is movement, mainly including respiratory movement and cardiac activity [10]. At present, most studies have mainly focused on the influence of respiratory movement on the treatment accuracy for thoracic tumors and have suggested many schemes to reduce the influence of respiratory movement, such as using Maximal intensity projection (MIP) images to contour the clinic target volume (CTV) and using active breathing control $(\mathrm{ABC})$ or deep inhalation breath holding $(\mathrm{DIBH})$ in the treatment process $[8,11,12]$. Nissen et al. conducted a group study on 319 breast cancer patients (144 patients in DIBH and 175 patients in free breathing (FB)), it found that the cardiac $\mathrm{V}_{20}, \mathrm{~V}_{40}$ and $\mathrm{D}_{\text {mean }}$ decreased from 7.8 to $2.3 \%, 3.4$ to $0.3 \%$, and 5.2 Gy to 2.7 Gy, respectively, for patients treated with DIBH compared with FB [13]. However, there are few studies on the influence of cardiac activity, and because cardiac activity is not consciously controlled like the respiratory movement, an effective method to reduce the impact of cardiac activity has not yet been proposed.

Our research group has attempted to find ways to reduce the effect of cardiac activity. Previously, we studied the impact of cardiac activity on the dose of the heart and its substructures, and the results indicated that the impact of cardiac activity on each cardiac structure, especially on the left ventricular myocardium (LVM), could not be ignored $[14,15]$. Unfortunately, no relevant solutions to this problem have been proposed.

On the basis of previous studies, according to the experience with solutions for respiratory movement, this study explored whether it is possible to reconstruct specific three-dimensional computed tomography (3DCT) images, i.e., generating MIP images, Average intensity projection (AIP) images and Minimum intensity projection (Min-IP) images, and contouring the heart, pericardium and LVM on these images to perform dose assessment to reduce the impact of cardiac activity, making the dose closer to the accumulated dose considered for cardiac activity.

\section{Materials and methods \\ Patients}

The data on electrocardiograph-gated four-dimensional computed tomography (ECG-gated 4DCT) for 22 patients based on breath-hold were analyzed retrospectively in this study; these patients were assessed from March 2015 to November 2016. There were 12 males and 10 females included among these patients, with ages ranging from 35 to 67 years, and the median age was 58 years. Esophageal tumors were evaluated in the present study. In addition, this study was approved by the Research Ethics Board of Shandong Cancer Hospital, and informed consent was obtained from all patients.

\section{Acquisition of 4DCT}

The ECG-gated 4DCT images of all patients were acquired with a Siemens dual-source CT (Siemens SOMATOM Definition, DER). Then, the acquired images were reconstructed via a $5 \%$ cardiac cycle, which were equally divided; the 20 phases cardiac cycle images were reconstructed $(0,5,10 \% \cdots \cdot . \cdot 95 \%)$ in this study, and all images were reconstructed at $0.75 \mathrm{~mm}$ slice thickness with an increment of $0.5 \mathrm{~mm}$. The image resolution was 512 * 512 , and the voxel size was $(0.69 * 0.69 * 0.5) \mathrm{mm}$.

\section{Acquisition of specific 3DCT images}

The 4DCT images were imported into MIM Maestro 6.8.2 (MIM) (MIM Software Inc., America) workstation to rebuild the required images. Three specific 3DCT images were reconstructed in this study: (1) MIP: The new CT value of a point in this study was defined as the CT value of the pixel with maximum density in 20 phases. (2) AIP: The new CT value of a point in this study was defined as the average CT value of all pixels in the 20 phases, which could be used to rebuild the conventional scanned images. (3) Min-IP: The new CT value of a point in this study was defined as the CT value of the pixel with minimum density in 20 phases.

\section{Contouring the heart, pericardium and LVM}

The heart, pericardium and LVM were contoured in all 20 phases (to acquire the dose of cardiac structures on each phase of 4DCT images conveniently, and the true accumulated dose can be assessed) and the specific 3DCT images (MIP, AIP and Min-IP images) by using MIM (Fig. 1). In this study, the upper boundary of the heart and pericardium was the top of the left atrium, and the lower boundary of the heart was the apex cordis. The lower boundary of the pericardium was defined as a loss of visual confirmation of the pericardium structure. The upper boundary of the LVM was the top 


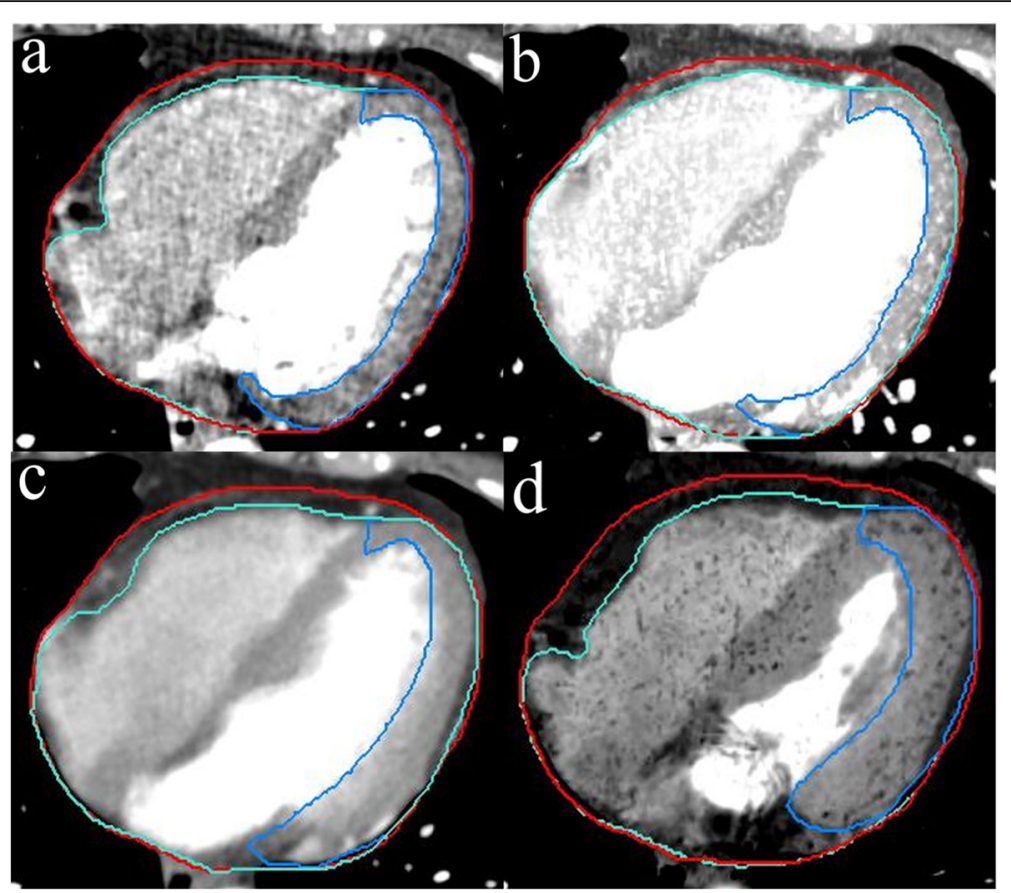

Fig. 1 Delineation of the heart, pericardium and left ventricular myocardium (LVM) in transverse section. (a) Delineation of the heart, pericardium and LVM on a phase image of 4DCT. (b) Delineation of the heart, pericardium and LVM on MIP images. (c) Delineation of the heart, pericardium and LVM on AIP images. (d) Delineation of the heart, pericardium and LVM on Min-IP images. The heart delineation is shown in cyan, the pericardium delineation is shown in red and the LVM delineation is shown in blue

of the left ventricle, and the lower boundary was the apex cordis. The interventricular septum was not included in this study. The window width/window level was $400 / 40 \mathrm{HU}$, and all delineations were performed by the same physician.

\section{Designing radiotherapy plans}

The treatment technology in this study was threedimensional conformal radiation therapy (3D-CRT). The planning target volume (PTV) was contoured on $0 \%$ phase image, and the radiotherapy plans were designed on the $0 \%$ phase image (Fig. 2). The prescribed dose of PTV was $60 \mathrm{~Gy}$ for all plans, the dose distribution met the requirement that $95 \%$ of the PTV received the prescribed dose, and the constraints of the organ at risk
(OARs) were as follows: total lung $\mathrm{V}_{20}<30 \%, \mathrm{~V}_{30}<20 \%$, maximum dose to the spinal cord $<45 \mathrm{~Gy}$, heart $\mathrm{V}_{30}<$ $40 \%$, and $\mathrm{V}_{40}<30 \%$.

\section{Dose accumulation and dose deformation}

To obtain the accumulated dose of 20 phases, we first transplanted the dose designed on $0 \%$ phase image to all phases of 4DCT to acquire the dose on all phases (this process is called the "duplication" in the MIM workstation), and then the dose accumulation of each phase was performed. The above procedures could be completed by the "Deformable Dose Accumulation" function provided in MIM. Through the above process, the accumulated dose that considered the cardiac activity could be acquired in this study (Fig. 3). The acquired accumulated

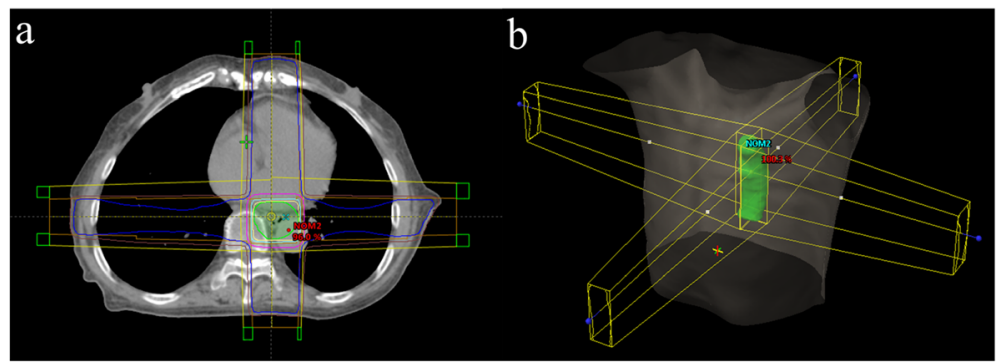

Fig. 2 An example image of the treatment plan. (a) Dose distribution of the 3D-CRT plan. (b) Setting of the radiation beams in 3D space 


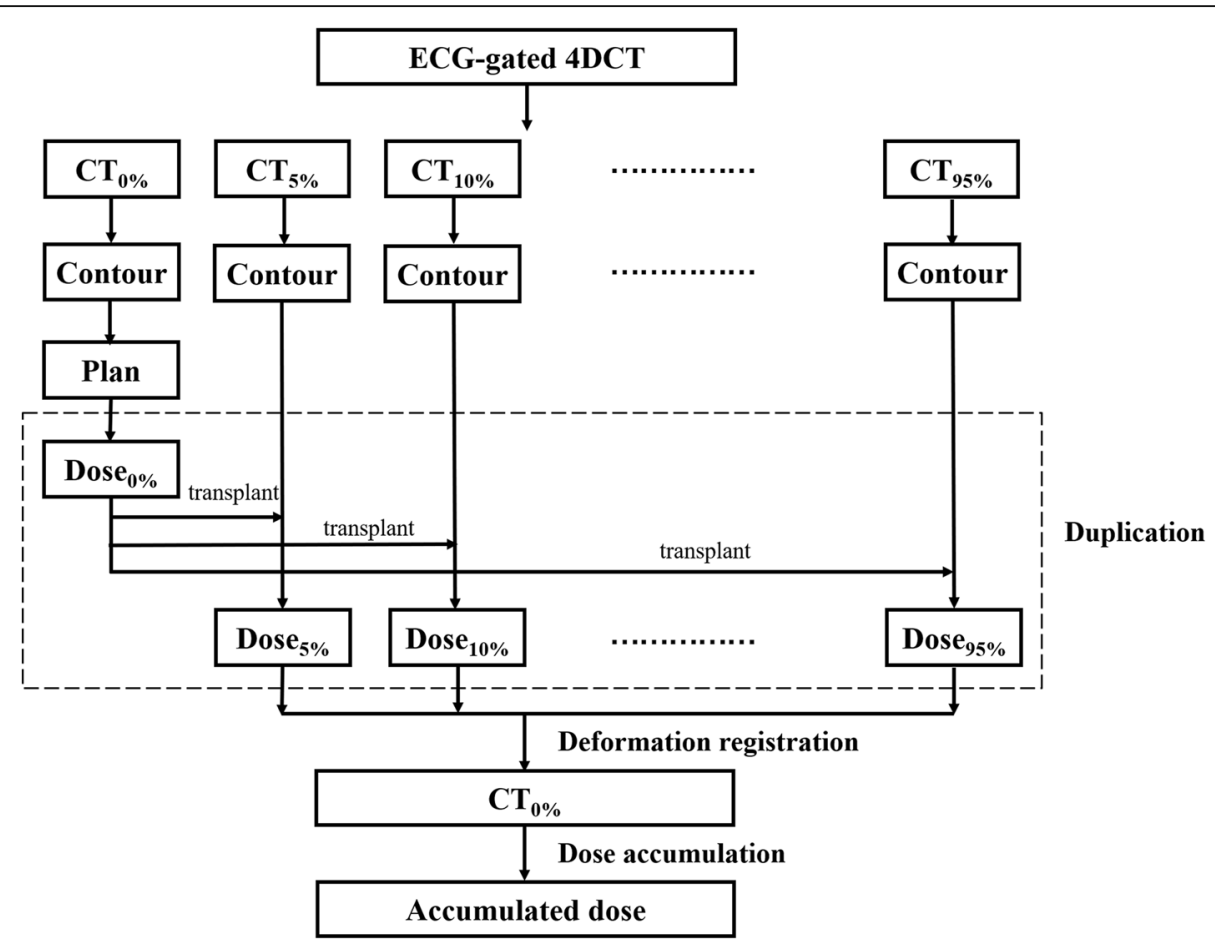

Fig. 3 Acquisition of the accumulated dose

dose was defined as the ground truth, and other results were compared to this dose to determine which result was closest to this dose.

The dose of the cardiac structures on specific 3DCT images (MIP, AIP and Min-IP images) was obtained by deforming the dose on $0 \%$ phase image to these images. Then, those results were compared to the accumulated dose. The deformation image registration (DIR) algorithm in this study was an intensity-based free-form DIR algorithm provided with the MIM software, which has been proven to have high accuracy [16].

\section{Data analysis}

To explore which of the three reconstructed doses was the most similar to the accumulated dose, the dose for the heart, pericardium and LVM on three specific 3DCT images and the accumulated dose calculated with 20 phases were analyzed. The dose-volume parameters mainly included $D_{\text {mean }}, V_{5}, V_{10}, V_{20}, V_{30}$ and $V_{40}$. In addition, $\Delta_{\text {(MIP }}$ vs. Accumulation) $/ \Delta_{(\text {AIP }}$ vs. Accumulation) $/$ $\Delta_{\text {(Min-IP vs. Accumulation })}=\mid$ Dose $\mathrm{MIP} / \mathrm{AIP} / \mathrm{Min}-\mathrm{IP}-$ Dose $_{\mathrm{Accu}}-$ mulation $\mid$, it was also used to describe volume differences, i.e., $\Delta_{\text {(MIP vs. Accumulation) }} / \Delta_{\text {(AIP vs. Accumulation) }} / \Delta_{\text {(Min-IP vs. }}$ Accumulation $)=\mid V_{X}$ MIP/AIP/Min-IP $-V_{X}$ Accumulation $\mid$, where $\mathrm{X}$ indicates $5,10,20,30$, and 40 .

\section{Statistical analyses}

All data were analyzed using SPSS v19.0 software (SPSS Inc., Chicago, IL), and the results were described as the mean \pm standard deviation $(\bar{x} \pm s)$. For comparisons of data between two groups, the independent samples $t$ test was used when the data showed a normal distribution and the variance was homogenous; otherwise, the Mann-Whitney U-test was used. The differences were considered statistically significant when $p<0.05$.

\section{Results}

Differences of $D_{\text {mean }}$ for the heart, pericardium and left ventricular myocardium after specific 3DCT image reconstruction and after accumulation

As shown in Table 1 and Fig. 4, for the reconstructed MIP image, the mean value of the differences in $D_{\text {mean }}$ were all less than $1.50 \mathrm{~Gy}$ for the heart, pericardium and LVM, which were compared with the $\mathrm{D}_{\text {mean }}$ after accumulation, and the pericardium had the smallest difference. The dose differences of pericardium and LVM in the MIP image were less than those in the AIP and MinIP images, which were all compared with the accumulated dose. For the reconstructed AIP and Min-IP images, the dose difference for the LVM was greater than those for the heart and pericardium, especially in the Min-IP images, the mean value of the difference for LVM reached to 3.20 Gy compared to that after accumulation, and the maximum difference was $5.66 \mathrm{~Gy}$. According to the statistical comparison, in the MIP image, only the dose difference for the heart was statistically significant $(p<0.05)$. In the AIP image, the dose differences for the heart and pericardium were statistically 
Table 1 Differences of $D_{\text {mean }}$ for the heart, pericardium and left ventricular myocardium after specific 3DCT image reconstruction and after accumulation

\begin{tabular}{llll}
\hline & Heart & Pericardium & Left ventricular myocardium \\
\hline Accumulation & $21.76 \pm 3.03$ & $21.87 \pm 2.59$ & $10.12 \pm 3.91$ \\
MIP & $23.01 \pm 2.24$ & $22.84 \pm 2.06$ & $10.05 \pm 3.31$ \\
AIP & $22.91 \pm 2.49$ & $22.96 \pm 2.17$ & $8.25 \pm 3.92$ \\
Min-IP & $22.67 \pm 3.07$ & $23.14 \pm 2.32$ & $7.16 \pm 4.52$ \\
$\Delta_{\text {(MIP vs. Accumulation) }}$ & $1.38 \pm 0.85$ & $1.04 \pm 0.65$ & $1.14 \pm 0.87$ \\
$\Delta_{\text {(AIP vs. Accumulation) }}$ & $1.21 \pm 0.63$ & $1.11 \pm 0.52$ & $1.93 \pm 1.20$ \\
$\Delta_{\text {(Min-IP vs. Accumulation) }}$ & $1.05 \pm 0.52$ & $1.27 \pm 0.65$ & $3.20 \pm 1.60$ \\
$P_{\text {(MIP vs. Accumulation) }}$ & 0.023 & 0.080 & 0.952 \\
$P_{\text {(AIP vs. Accumulation) }}$ & 0.033 & 0.032 & 0.122 \\
$P_{\text {(Min-IP vs. Accumulation) }}$ & 0.116 & 0.019 & 0.002 \\
\hline
\end{tabular}

significant $(p<0.05)$. In the Min-IP image, the dose differences for the pericardium and LVM were all statistically significant $(p<0.05)$.

\section{Differences of dose-volume parameters for the heart after specific 3DCT image reconstruction and after accumulation}

As shown in Table 2, the difference between cardiac dosevolume parameters (such as $\mathrm{V}_{5}, \mathrm{~V}_{10}, \mathrm{~V}_{20}, \mathrm{~V}_{30}$ and $\mathrm{V}_{40}$ ) in MIP, AIP, Min-IP images and those after accumulation were similar, and the average value of the difference were all less than $4 \%$. In addition, for the heart, the average values of the differences for $\mathrm{V}_{5}, \mathrm{~V}_{10}, \mathrm{~V}_{20}$ and $\mathrm{V}_{40}$ in MIP, AIP, and Min-IP images were no more than $3 \%$ compared to those after accumulation, and this difference was not statistically significant after statistical analysis $(p>0.05)$.

\section{Differences of dose-volume parameters for the} pericardium after specific 3DCT image reconstruction and after accumulation

The differences between the dose-volume parameters (such as $\mathrm{V}_{5}, \mathrm{~V}_{10}, \mathrm{~V}_{20}, \mathrm{~V}_{30}$ and $\mathrm{V}_{40}$ ) for the pericardium in the MIP, AIP, Min-IP images and those parameters after accumulation were shown in Table 3 . The results showed that regardless of which of the three reconstruction methods was adopted, the differences of $V_{30}$ and $\mathrm{V}_{40}$ for the pericardium in the reconstructed images were statistically significant compared to those after accumulation $(p<0.05)$. Among these values, the mean value of the difference between the $V_{30}$ for the pericardium in the Min-IP image and the accumulated dose reached $4.51 \%$, and the maximum difference reached $10.07 \%$. However, there was no statistically significant difference for $\mathrm{V}_{5}, \mathrm{~V}_{10}$ and $\mathrm{V}_{20}$ of the pericardium in all reconstructed images compared to these parameters after accumulation $(p>0.05)$.

\section{Differences of dose-volume parameters for the left ventricular myocardium after specific 3DCT image reconstruction and after accumulation}

As shown in Table 4, for the LVM, among all the reconstructed images, the difference of dose-volume parameters (such as $\mathrm{V}_{5}, \mathrm{~V}_{10}, \mathrm{~V}_{20}, \mathrm{~V}_{30}$ and $\mathrm{V}_{40}$ ) on the MIP image was the smallest compared to those after

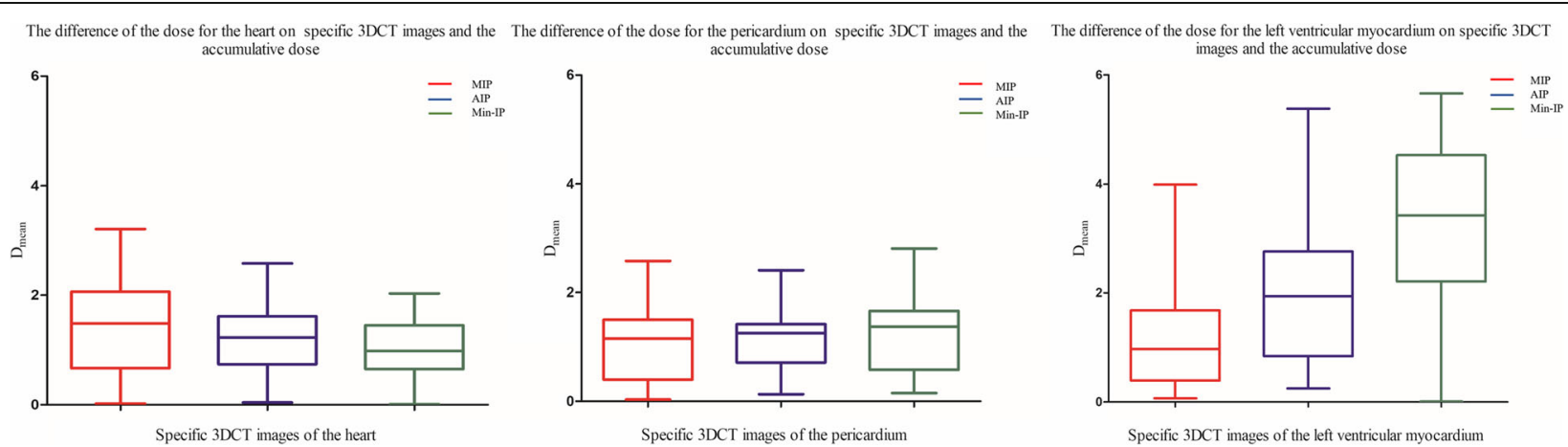

Fig. 4 Differences in the dose for the heart, pericardium and left ventricular myocardium on specific 3DCT images and the accumulated dose. The difference in the dose on the MIP image is shown in red, the difference in the dose on the AIP image is shown in blue, and the difference in the dose on the Min-IP image is shown in green 
Table 2 Differences of dose-volume parameters for the heart after specific 3DCT image reconstruction and after accumulation

\begin{tabular}{llllll}
\hline & $V_{5}$ & $V_{10}$ & $V_{20}$ & $V_{30}$ & $V_{40}$ \\
\hline Accumulation & $69.33 \pm 8.02$ & $56.38 \pm 6.42$ & $48.88 \pm 6.34$ & $42.31 \pm 6.35$ & $12.31 \pm 5.73$ \\
MIP & $70.46 \pm 6.03$ & $58.26 \pm 5.43$ & $51.45 \pm 5.32$ & $45.82 \pm 5.21$ & $13.53 \pm 6.22$ \\
AIP & $70.81 \pm 6.45$ & $58.25 \pm 6.16$ & $51.42 \pm 6.08$ & $45.71 \pm 6.04$ & $12.72 \pm 6.14$ \\
Min-IP & $71.32 \pm 7.55$ & $58.34 \pm 7.76$ & $51.43 \pm 7.77$ & $45.70 \pm 7.70$ & $11.51 \pm 6.00$ \\
$\Delta_{\text {(MIP vs. Accumulation) }}$ & $2.38 \pm 1.53$ & $2.17 \pm 1.23$ & $2.67 \pm 1.69$ & $3.51 \pm 1.81$ & $1.23 \pm 1.00$ \\
$\Delta_{\text {(AIP vs. Accumulation) }}$ & $2.22 \pm 1.11$ & $1.89 \pm 0.99$ & $2.53 \pm 1.02$ & $3.40 \pm 1.15$ & $0.83 \pm 0.83$ \\
$\Delta_{\text {(Min-IP vs. Accumulation) }}$ & $2.04 \pm 1.29$ & $2.15 \pm 1.72$ & $2.71 \pm 1.86$ & $3.45 \pm 2.12$ & $1.47 \pm 1.14$ \\
$P_{\text {(MIP vs. Accumulation) }}$ & 0.258 & 0.205 & 0.078 & 0.018 & 0.250 \\
P (AIP vs. Accumulation) & 0.406 & 0.152 & 0.054 & 0.029 & 0.639 \\
P (Min-IP vs. Accumulation) & 0.809 & 0.231 & 0.116 & 0.054 & 0.467 \\
\hline
\end{tabular}

accumulation, and the average value of these difference were less than $4 \%$; the differences were not statistically significant $(p>0.05)$. However, the difference of $\mathrm{V}_{5}, \mathrm{~V}_{10}$, $\mathrm{V}_{20}, \mathrm{~V}_{30}$ and $\mathrm{V}_{40}$ for LVM in the Min-IP image was the largest compared to that after accumulation, and the mean value of the difference for $V_{10}$ reached $10.66 \%$, the maximum value reached $19.49 \%$, and the differences were statistically significant $(p<0.05)$. There was a statistically significant difference between the $\mathrm{V}_{40}$ of LVM in the AIP image and that after accumulation $(p<0.05)$.

\section{Discussion}

The feasibility of using MIP images to perform CTV assessments is usually discussed by using static parameters, such as the volume or morphology of CTV, however, in this study, the feasibility of using MIP images to evaluate the dose of cardiac structures was innovatively expounded from the perspective of dosimetry, which has a great significance for the dose assessment and the protection of the heart in clinical practice. The results of this study showed that there was a lesser difference in the dose for the heart, pericardium and LVM in the MIP image compared to the dose after accumulation, so the MIP image could be used for the clinical dose evaluation of cardiac structures.

Organ movement has always been an important factor affecting the improvement of radiotherapy accuracy, and some studies have shown that respiratory movement has a serious influence on the radiotherapy effect for thoracic tumors [17, 18]. Giraud et al. mentioned that respiratory movements on the order of $1 \mathrm{~cm}$ were unacceptable in radiotherapy [19]. To reduce the impact of respiratory movement, DIBH has been applied maturely, this technology was also adopted in this study to better analyze cardiac movement. Simonetto et al. conducted a prospective study of 89 patients with left breast cancer and the results indicated that with the use of DIBH, mean cardiac doses were reduced by $35 \%$ compared to $\mathrm{FB}$, and the mean expected years of life lost due to radiation-induced ischemic heart disease mortality were 0.11 years in FB, and 0.07 years in DIBH [20]. Ledsom et al. analyzed 30 patients with left breast cancer also showed that all patients achieved decreased $V_{30}$ and mean cardiac dose using DIBH [12].

However, Kataria et al. proposed that in addition to respiratory movement, cardiac activity is also an important

Table 3 Differences of dose-volume parameters for the pericardium after specific 3DCT image reconstruction and after accumulation

\begin{tabular}{llllll}
\hline & $V_{5}$ & $V_{10}$ & $V_{20}$ & $V_{30}$ & $V_{40}$ \\
\hline Accumulation & $68.37 \pm 6.76$ & $56.85 \pm 7.79$ & $49.16 \pm 7.75$ & $42.40 \pm 7.67$ & $10.86 \pm 5.58$ \\
MIP & $69.34 \pm 5.57$ & $57.42 \pm 5.00$ & $50.72 \pm 4.78$ & $45.11 \pm 4.69$ & $14.16 \pm 6.24$ \\
AIP & $69.74 \pm 5.72$ & $57.85 \pm 5.27$ & $51.18 \pm 5.04$ & $45.57 \pm 4.93$ & $13.98 \pm 6.44$ \\
Min-IP & $70.73 \pm 5.93$ & $58.77 \pm 5.75$ & $52.04 \pm 5.56$ & $46.43 \pm 5.45$ & $13.82 \pm 6.75$ \\
$\Delta_{\text {(MIP vs. Accumulation) }}$ & $1.69 \pm 1.01$ & $2.91 \pm 2.22$ & $3.37 \pm 2.34$ & $3.91 \pm 2.39$ & $3.31 \pm 1.44$ \\
$\Delta_{\text {(AIP vs. Accumulation) }}$ & $1.77 \pm 0.87$ & $2.76 \pm 2.10$ & $3.38 \pm 2.07$ & $3.95 \pm 2.26$ & $3.12 \pm 1.37$ \\
$\Delta_{\text {(Min-IP vs. Accumulation) }}$ & $2.48 \pm 1.38$ & $3.23 \pm 2.07$ & $3.86 \pm 2.14$ & $4.51 \pm 2.42$ & $2.96 \pm 1.56$ \\
$P_{\text {(MIP vs. Accumulation) }}$ & 0.349 & 0.348 & 0.152 & 0.041 & 0.012 \\
P (AIP vs. Accumulation) & 0.432 & 0.205 & 0.082 & 0.018 & 0.015 \\
P (Min-IP vs. Accumulation) & 0.545 & 0.133 & 0.051 & 0.012 & 0.021 \\
\hline
\end{tabular}


Table 4 Differences of dose-volume parameters for the left ventricular myocardium after specific 3DCT image reconstruction and after accumulation

\begin{tabular}{llllll}
\hline & $V_{5}$ & $V_{10}$ & $V_{20}$ & $V_{30}$ & $V_{40}$ \\
\hline Accumulation & $36.82 \pm 12.55$ & $25.66 \pm 10.47$ & $19.36 \pm 9.75$ & $13.87 \pm 8.39$ & $3.50 \pm 3.71$ \\
MIP & $35.73 \pm 10.50$ & $25.58 \pm 9.32$ & $19.94 \pm 8.62$ & $15.44 \pm 7.74$ & $2.79 \pm 2.79$ \\
AIP & $31.91 \pm 14.15$ & $20.55 \pm 11.47$ & $15.25 \pm 9.89$ & $10.74 \pm 8.22$ & $1.57 \pm 2.82$ \\
Min-IP & $28.62 \pm 17.29$ & $15.81 \pm 13.97$ & $10.79 \pm 11.59$ & $7.13 \pm 9.76$ & $1.18 \pm 2.76$ \\
$\Delta_{\text {(MIP vs. Accumulation) }}$ & $3.59 \pm 2.73$ & $3.45 \pm 1.88$ & $3.27 \pm 1.72$ & $3.01 \pm 1.89$ & $1.13 \pm 1.40$ \\
$\Delta_{\text {(AIP vs. Accumulation) }}$ & $5.77 \pm 3.01$ & $5.19 \pm 2.59$ & $4.35 \pm 2.60$ & $3.56 \pm 2.61$ & $2.01 \pm 2.15$ \\
$\Delta_{\text {(Min-IP vs. Accumulation) }}$ & $9.28 \pm 4.29$ & $10.66 \pm 5.23$ & $9.48 \pm 5.31$ & $7.83 \pm 4.63$ & $2.44 \pm 2.32$ \\
$P_{\text {(MIP vs. Accumulation) }}$ & 0.981 & 0.961 & 0.944 & 0.970 & 0.742 \\
P (AIP vs. Accumulation) & 0.546 & 0.692 & 0.967 & 0.121 & 0.039 \\
P (Min-IP vs. Accumulation) & 0.006 & 0.002 & 0.002 & 0.004 & 0.001 \\
\hline
\end{tabular}

factor affecting the accuracy of treatment [10]. There have been few previous studies focusing on cardiac activity, one of the important reasons is that the heart moves faster and its morphological variation is hard to capture. With the improvement of CT scanning speed and CT image quality, and the emergence of dual-source CT, which makes it possible to obtain a clear ECG-gated 4DCT image, the morphological variations of cardiac structures in the entire cardiac cycle can be displayed truly and clearly, which lays a foundation for studying the impact of cardiac activity on cardiac dose and proposing relevant solutions. This study made good use of this technology and explored a solution to reduce the impact of cardiac activity on cardiac structures.

According to previous solutions for respiratory movement, it may be beneficial to contour organs on the specific 3DCT images. René et al. reported that MIPs were a reliable clinical tool for generating internal target volumes (ITVs) from 4DCT data sets [21]. Muirhead et al. also suggested that the MIP images could be used for delineation in Stage I non-small cell lung cancer (NSCLC) tumors to prevent the under-treatment of disease [22]. This study was conducted from the perspective of dosimetry, if the accumulated dose of the heart, pericardium and LVM in 20 phases was taken as a reference, among the reconstructed MIP, AIP and Min-IP images, was there an image on which the dose for the heart, pericardium and LVM was the closest to the accumulated dose, which could be used for the clinical delineation of cardiac structures?

For $D_{\text {mean }}$, the mean value of the difference between the $D_{\text {mean }}$ on the MIP image and the $D_{\text {mean }}$ after accumulation for the heart, pericardium and LVM were all less than $1.50 \mathrm{~Gy}$, especially for the pericardium and LVM, the difference was the smallest among the three reconstructed images, and this difference was not statistically significant after statistical comparison. This result indicated that MIP images were more suitable for assessing the dose of cardiac structures, especially for LVM with large morphological variations, MIP images have shown irreplaceable advantages. On the MIP image, the difference between the $\mathrm{D}_{\text {mean }}$ and the accumulated dose for the pericardium was the smallest, which also proved the conclusion of our previous study, namely, that the pericardium contour had better stability, and the pericardium contour could be considered as an OAR to protect the heart in clinical practice [15].

To better compare the ability of dose assessment for each specific 3DCT image, we selected a number of dose-volume parameters, including $\mathrm{V}_{5}$ and $\mathrm{V}_{10}$, which represent the low-dose region, as well as the common parameters used for clinical evaluation, $\mathrm{V}_{30}$ and $\mathrm{V}_{40}$. The results showed that for the heart and pericardium, these three reconstructed images (MIP, AIP and Min-IP images) had a similar ability for dose assessment, especially for the low-dose region, $V_{5}, V_{10}$ and $V_{20}$, the difference between these parameters for the heart and pericardium in all reconstructed images was not significant compared to those after accumulation. However, for the LVM, the advantage of using the MIP image for dose assessment was more obvious. The difference between all dosevolume parameters for the LVM in the MIP image and those parameters after accumulation was the smallest, and this difference was not statistically significant. Additionally, the Min-IP image could not be used to evaluate the dose of the LVM because of the large dose difference. All the above results indicate that the MIP image had a good ability for dose assessment and could be recommended for contouring the cardiac structures and performing the dose assessment. This study was based on 3D-CRT, a static approach, and further research could be performed in dynamic therapies, such as intensity modulated radiation therapy (IMRT) or volumetric intensity modulated arc therapy (VMAT). For example, future studies can contour the cardiac structures directly on MIP images and deform these structures to planning 
CT to perform the dose limitation, follow up patients who received the treatment, and compare these patients to those who received traditional assessment to further demonstrate that using the MIP images to contour cardiac structures can achieve effective protection for the cardiac structures of patients with esophageal cancer.

In addition, theoretically, AIP images can be considered as plain CT images, which can be used for designing radiotherapy plans. However, according to the results of this study, regardless of the heart, pericardium or LVM, there were some differences between the dose parameters in the AIP image and the dose parameters after accumulation, which showed that the cardiac activity has caused the difference in dose assessment for cardiac structures. The requirement to solve this problem was urgent, which also indicated the significance of this study.

\section{Conclusions}

This study reconstructed the MIP, AIP and Min-IP images and contoured the heart, pericardium and LVM on these images to perform the dose assessment, compared with the dose parameters which after accumulation showed that, there was a smallest difference between the dosimetry parameters on the MIP image and the corresponding parameters after dose accumulation for cardiac structures. Thus, it is recommended to use the MIP image for the delineation and the dose assessment of cardiac structures in clinical practice.

\section{Abbreviations \\ 3DCT: Three-dimensional computed tomography; 4DCT: Four- dimensional computed tomography; ABC: Active breathing control; AIP: Average intensity projection; CTV: Clinic target volume; DIBH: Deep inhalation breath holding; DIR: Deformation image registration; ECG-gated 4DCT: Electrocardiograph- gated 4DCT; FB: Free breathing; IMRT: Intensity modulated radiation therapy; ITV: Internal target volume; LVM: Left ventricular myocardium; Min- IP: Minimum intensity projection; MIP: Maximal intensity projection; NSCLC: Non-small cell lung cancer; OAR: Organ at risk; PTV: Planning target volume; RIHD: Radiation-induced heart disease; VMAT: Volumetric intensity modulated arc therapy}

\section{Acknowledgements}

Not applicable.

\section{Authors' contributions}

YY and GGZ planned this retrospective study. TY was responsible for data collection and statistical analysis and drafted the manuscript. GGZ, SM reviewed and commented on the results of the study. All authors read and approved the final manuscript.

\section{Funding}

This study was supported by the Shandong key research and development programs (No. 2018GSF118048), National key research and development programs of China (No. 2017YFC0113202), National Natural Science Foundation of China (No. 81472811) and Shandong key research and development programs (No. 2018GSF118006).

Availability of data and materials Not applicable.

\section{Ethics approval and consent to participate}

This study was approved by the Research Ethics Board of the Shandong Cancer Hospital, and informed consent was obtained from all the patients.

\section{Consent for publication}

Not applicable.

\section{Competing interests}

The authors declare that they have no competing interests.

\section{Author details}

'Shandong Cancer Hospital and Institute, Shandong First Medical University and Shandong Academy of Medical Sciences, Jinan, China. ${ }^{2}$ School of Nuclear Science and Technology, University of South China, Hengyang, China

Received: 17 June 2019 Accepted: 19 December 2019

Published online: 27 December 2019

\section{References}

1. Taylor CW, Brønnum D, Darby SC, et al. Cardiac dose estimates from Danish and Swedish breast cancer radiotherapy during 1977-2001[J]. Radiother Oncol. 2011;100(2):176-83.

2. Joseph N, Mcwilliam A, Kennedy J, et al. Post-treatment lymphocytopenia, integral body dose and overall survival in lung cancer patients treated with radical radiotherapy[]]. Radiother Oncol. 2019;135:115-9.

3. Zhu Q, Kirova YM, Cao L, et al. Cardiotoxicity associated with radiotherapy in breast cancer: a question-based review with current literatures[J]. Cancer Treat Rev. 2018:68:9-15.

4. Kole TP, Aghayere O, Kwah J, et al. Comparison of heart and coronary artery doses associated with intensity-modulated radiotherapy versus threedimensional conformal radiotherapy for distal esophageal cancer[]]. Int J Radiat Oncol Biol Phys. 2012;83:1580-6.

5. Madan R, Benson R, Sharma DN, et al. Radiation induced heart disease: pathogenesis, management and review literature[J]. J Egypt Natl Canc Inst 2015;27(4):187-93.

6. Duane FK, McGale P, Brønnum D, et al. Cardiac structure doses in women irradiated for breast Cancer in the past and their use in epidemiological studies[J]. Pract Radiat Oncol. 2019;9(3):158-71.

7. Evans ES, Prosnitz RG, Yu X, et al. Impact of patient-specifc factors, irradiated left ventricular volume, and treatment set-up errors on the development of myocardial perfusion defects after radiation therapy for left-sided breast cancer[]]. Int J Radiat Oncol Biol Phys. 2006;66(4):1125-34.

8. Mast ME, Van Kempen-Harteveld L, Heijenbrok MW, et al. Left-sided breast cancer radiotherapy with and without breath-hold: does IMRT reduce the cardiac dose even further? [J]. Radiother Oncol. 2013:108(2):248-53.

9. Shah C, Badiyan S, Berry S, et al. Cardiac dose sparing and avoidance techniques in breast cancer radiotherapy[]]. Radiother Oncol. 2014; 112(1):9-16.

10. Kataria T, Bisht SS, Gupta D, et al. Quantification of coronary artery motion and internal risk volume from ECG gated radiotherapy planning scans[]]. Radiother Oncol. 2016;121:59-63.

11. Lee S, Zheng Y, Podder T, et al. Tumor localization accuracy for highprecision radiotherapy during active breath-hold[]]. Radiother Oncol. 2019; 137:145-52

12. Ledsom D, Reilly AJ, Probst H. Assessment of deep inspiration breath hold (DIBH) amplitude and reduction in cardiac dose in left breast cancer patients[]]. Radiogr (Lond). 2018;24(2):98-103.

13. Nissen HD, Appelt AL. Improved heart, lung and target dose with deep inspiration breath hold in a large clinical series of breast cancer patients[]]. Radiother Oncol. 2013;106(1):28-32.

14. Ying $T$, Yong $Y$, Pinjing $C$, et al. Quantification of variation in dose-volume parameters for the heart, pericardium and left ventricular myocardium. during thoracic tumor radiotherapy[J]. J Radiat Res. 2018:59(4):462-8.

15. Ying $T$, Yong $Y$, Jie $L$, et al. Quantification of heart, pericardium, and left ventricular myocardium movements during the cardiac cycle for thoracic tumor radiotherapy[]]. Onco Targets Ther. 2018;11:547-54.

16. Piper J. Evaluation of an intensity-based free-form deformable registration algorithm[J]. Med Phys. 2007;34:2353-4. 
17. Jang SS, Huh GJ, Park SY, et al. The impact of respiratory gating on lung dosimetry in stereotactic body radiotherapy for lung cancer[J]. Phys Med. 2014;30(6):682-9.

18. Shimizu S, Shirato $H$, Kagei $\mathrm{K}$, et al. Impact of respiratory movement on the computed tomographic images of small lung tumors in three-dimensional (3D) radiotherapy[J]. Int J Radiat Oncol Biol Phys. 2000;46(5):1127-33.

19. Giraud $\mathrm{P}$, Morvan $E$, Claude $L$, et al. Respiratory gating techniques for optimization of lung Cancer radiotherapy[]]. J Thorac Oncol. 2011;6(12): 2058-68.

20. Simonetto C, Eidemüller M, Gaasch A, et al. Does deep inspiration breathhold prolong life? Individual risk estimates of ischaemic heart disease after breast cancer radiotherapy[]]. Radiother Oncol. 2019;131(2):202-7.

21. Underberg RW, Lagerwaard FJ, Slotman BJ, et al. Use of maximum intensity projections (MIP) for target volume generation in $4 \mathrm{DCT}$ scans for lung cancer[J]. Int J Radiat Oncol Biol Phys. 2005:63(1):253-60.

22. Muirhead $R, M c n e e ~ S G$, Featherstone $C$, et al. Use of maximum intensity projections (MIPs) for target outlining in $4 \mathrm{DCT}$ radiotherapy planning[J]. J Thorac Oncol. 2008;3(12):1433-8.

\section{Publisher's Note}

Springer Nature remains neutral with regard to jurisdictional claims in published maps and institutional affiliations.

Ready to submit your research? Choose BMC and benefit from:

- fast, convenient online submission

- thorough peer review by experienced researchers in your field

- rapid publication on acceptance

- support for research data, including large and complex data types

- gold Open Access which fosters wider collaboration and increased citations

- maximum visibility for your research: over $100 \mathrm{M}$ website views per year

At BMC, research is always in progress.

Learn more biomedcentral.com/submissions 